\title{
Did the Gravity Probe B Actually Prove the Einstein's General Relativity Theory?
}

\author{
Jaroslav Hynecek ${ }^{1}$ \\ ${ }^{1} 2013$ Isetex, Inc., 905 Pampa Drive, Allen, TX, USA \\ Correspondence: Jaroslav Hynecek, 2013 Isetex, Inc., 905 Pampa Drive, Allen, TX 75013, USA. E-mail: \\ jhynecek@netscape.net
}

Received: January 14, 2013 Accepted: February 24, 2013 Online Published: May 2, 2013

doi:10.5539/apr.v5n3p90

URL: http://dx.doi.org/10.5539/apr.v5n3p90

\begin{abstract}
It has been shown in an earlier publication (Hynecek, 2013) that the frame dragging effect due to the Earth's rotation, which is claimed that the Gravity Probe B (GP-B) has detected, cannot exist. In this paper it is shown that the Geodetic Precession, which the GP-B has also measured, and is typically calculated from the Einstein's General Relativity Theory (GRT), can also be calculated from a different metric. When the results of calculations are compared side by side the differences are very small, but it becomes clear that the GRT actually may not be providing the correct derivation of formula for this effect. The one reason among several others is due to the fact that the GRT derivation, as is shown in this paper, also leads to the derivation of the classical Kepler's third law for circular orbits, which is easily derived from the Newtonian physics, and should therefore hold true only in the flat space-time geometry.
\end{abstract}

Keywords: Gravity Probe B, Metric Theory of Gravity, curved space-time metric, Schwarzschild metric, Christoffel coefficients, covariant derivative, Kepler's third law for circular orbits, geodetic precession

\section{Introduction}

Since the mathematicians have invaded the theory of relativity, I do not understand it myself anymore.

Albert Einstein

The geodetic precession is an effect that is predicted from the metric theory of gravity (MTG) and also from the GRT, which is the subset of MTG. The reason for this precession is the curved space-time. The precession calculation is actually a nice exercise in geometry that is performed in the next section of this paper for a new metric derived earlier by Hynecek (2012). Once the calculation procedure is established and the prediction compared with experimental results of GP-B, another calculation, along the same lines, is made using the Schwarzschild metric. Both theoretical predictions are then compared with observations and it is concluded that the GP-B has actually proved the correctness of the new MTG metric rather than the Schwarzschild metric. There are several reasons for this conclusion, as is described in detail in the following sections of the paper, with the main one being the unexpected discovery of a hidden error in the GRT calculations that falsely forces an agreement between the theory and experiment.

\section{Mathematical Background}

For the derivation of geodetic precession it will be necessary to know the metric and from that the Christoffel coefficients. The metric line element for the space-time of the centrally gravitating body that does not rotate, and as expressed in the standard spherical coordinates, was derived previously in MTG by Hynecek (2012):

$$
d s^{2}=g_{00}(c d t)^{2}-d \rho^{2}-\rho^{2} g_{00} d \Omega^{2}
$$

where the metric variables have the usual definitions: $d \rho=g_{00}{ }^{-1 / 2} d r, g_{00}=\exp \left(-R_{s} / \rho\right), d \Omega^{2}=d \vartheta^{2}+\sin ^{2} \vartheta d \phi^{2}$, and where: $R_{s}=2 \kappa M / c^{2}$ is the Schwarzschild radius. It was also shown previously that this metric agrees well with the famous four tests of the GRT and that it does not lead to any pathology such as the event horizon and Black Holes. The Christoffel coefficients that will be needed for the derivation of geodetic precession were also derived for this metric earlier by Hynecek (2007) and are as follows: 


$$
\begin{gathered}
\Gamma_{00}^{r}=\frac{\phi_{c}}{\rho} e^{-3 \phi_{c}} \\
\Gamma_{0 r}^{0}=-\Gamma_{r r}^{r}=\frac{\phi_{c}}{\rho} e^{\phi_{c}} \\
\Gamma_{\vartheta \vartheta}^{r}=\Gamma_{\phi \phi}^{r}=-\rho\left(1+\phi_{c}\right) e^{-3 \phi_{c}} \\
\Gamma_{r \phi}^{\phi}=\Gamma_{r \vartheta}^{\vartheta}=\frac{1+\phi_{c}}{\rho} e^{\phi_{c}}
\end{gathered}
$$

In these formulas the parameter $\phi_{c}$, which is the normalized potential and should not be confused with the angle $\phi$, was introduced to simplify notation and is equal to: $\phi_{c}=R_{s} / 2 \rho$. The notation for the metric signature was also modified to read: $(0,-r,-\vartheta,-\phi)$. In addition it was assumed that without the loss of generality the satellite is orbiting Earth in the equatorial plane. In actuality the satellite that has carried four gyroscopes has orbited in the precise polar orbit. This modification is not important for the results of this paper's derivations and it is only a matter of calculation convenience. Of course, there are many subtle effects that can have a significant influence on the experimental results and most likely would mask the frame dragging phenomenon if such an effect had actually existed. These are, for example, the imperfect shape of Earth, which is not precisely spherical, the tidal effects, the gyroscope powering patches, and so on (NASA, 2008, 2011). All these problems are discussed in the relevant literature (Wikipedia, 2013) and are not the subject of this paper.

Because this metric and the related Christoffel coefficients are new and have not been previously used for this type of calculations this work was exciting for the author with a degree of anxiety and anticipation whether the calculation results would actually agree with the experimental results and would thus prove the correctness of the new MTG metric and the corresponding curved space-time that has no event horizon and no Black Hole pathologies.

The geodetic precession is calculated by transporting the spin vector $S$ of a gyroscope in a parallel transport fashion along the satellite's orbit. This means that the covariant derivative of this vector along this trajectory must be zero. This is usually written in the tensor calculus coordinate notation according to Synge and Schild (1949) as follows:

$$
\frac{d S^{i}}{d \tau}+\Gamma_{j k}^{i} S^{j} u^{k}=0
$$

The variables $u^{k}$ are the components of the trajectory velocity 4-vector defined below:

$$
\begin{gathered}
u^{0}=\frac{c d t}{d \tau} \\
u^{\phi}=\frac{d \phi}{d \tau}
\end{gathered}
$$

The other two velocity vector components are zero, because the orbital radius and the angle $\vartheta$ are considered constant. Decomposing the formula in Equation 6 into its components and always keeping in mind that the orbital radius is constant this equation results in the following formulas:

$$
\begin{gathered}
\frac{d S^{0}}{d \tau}=-\Gamma_{0 r}^{0} u^{0} S^{r} \\
\frac{d S^{r}}{d \tau}=-\Gamma_{00}^{r} u^{0} S^{0}-\Gamma_{\phi \phi}^{r} u^{\phi} S^{\phi} \\
\frac{d S^{\phi}}{d \tau}=-\Gamma_{r \phi}^{\phi} u^{\phi} S^{r}
\end{gathered}
$$

Equation for the spin vector component related to $\vartheta$ is not important because this component is a constant of motion. Differentiating Equation 10 with respect to $\tau$ and substituting into the result of differentiation expressions for the Christoffel coefficients yields the following: 


$$
\frac{d^{2} S^{r}}{d \tau^{2}}=-S^{r} e^{-2 \phi_{c}}\left(\left(1+\phi_{c}\right)^{2}\left(u^{\phi}\right)^{2}-\frac{\phi_{c}^{2}}{\rho^{2}}\left(u^{0}\right)^{2}\right)
$$

In the next step of derivation it is necessary to find the velocity vector components appearing in Equation 12 . This is accomplished by a covariant differentiation of the velocity vector $u$, again along the orbit trajectory, where for the radial component of this vector holds the following:

$$
\frac{d u^{r}}{d \tau}=-\Gamma_{00}^{r}\left(u^{0}\right)^{2}-\Gamma_{\phi \phi}^{r}\left(u^{\phi}\right)^{2}=0
$$

The second equation for the velocity vector components is obtained from the metric considering that $d s=c d \tau$ :

$$
c^{2}=e^{-2 \phi_{c}}\left(u^{0}\right)^{2}-\rho^{2} e^{-2 \phi_{c}}\left(u^{\phi}\right)^{2}
$$

Solving this set of two equations for the two unknowns and again after the substitution for the Christoffel coefficients the results become as follows:

$$
\begin{gathered}
\left(u^{0}\right)^{2}=\left(1+\phi_{c}\right) c^{2} e^{2 \phi_{c}} \\
\left(u^{\phi}\right)^{2}=\frac{\phi_{c} c^{2}}{\rho^{2}} e^{2 \phi_{c}}
\end{gathered}
$$

The formula in Equation 12 represents a harmonic motion with the frequency $\omega_{s \tau}$. By substituting into this formula the expressions from Equations 15 and 16 the result for this frequency, which is the proper frequency, becomes:

$$
\omega_{s \tau}^{2}=e^{-2 \phi_{c}}\left(\left(1+\phi_{c}\right)^{2}\left(u^{\phi}\right)^{2}-\frac{\phi_{c}^{2}}{\rho^{2}}\left(u^{0}\right)^{2}\right)=\frac{c^{2}}{\rho^{2}} \phi_{c}\left(1+\phi_{c}\right)
$$

In the next step this frequency will be observed by a distant observer. This is indicated by a change in the subscript: $\tau \Rightarrow t$. The frequency transformation formula can be derived from Equation 15 resulting in the following:

$$
\omega_{s t}^{2}=\omega_{s \tau}^{2}\left(1+\phi_{c}\right)^{-1} e^{-2 \phi_{c}}=\frac{c^{2}}{\rho^{2}} \phi_{c} e^{-2 \phi_{c}}
$$

This frequency will now be compared with the reference orbital frequency that could be measured, for example, by a frequency counter representing clocks onboard of the satellite. The satellite orbital frequency observed by a distant observer is found from the Kepler's third law for circular orbits as published by Hynecek (2010), or alternately as can be derived from the formula: $\omega_{k t}=c u^{\phi} / u^{0}$. To find the corresponding satellite onboard frequency it is necessary to use the frequency transformation formula: $\omega_{\tau}=\omega_{t} \exp \left(\phi_{c}\right)$, which is the well known gravitational red shift formula.The detail derivation of this relation is described in the Appendix. Therefore, for the orbital frequency onboard of the satellite that determines the periodic observations of the reference star and is expressed in terms of the orbital frequency $\omega_{t 0}$ observed by a distant observer it must hold:

$$
\omega_{t 0}^{2}=\omega_{k t}^{2} \frac{\omega_{\tau}^{2}}{\omega_{t}^{2}}=\frac{c^{2}}{\rho^{2}} \frac{\phi_{c}}{1+\phi_{c}} e^{2 \phi_{c}}
$$

Dividing Equation 18 by Equation 19 results in the formula for the spin vector frequency normalized to the satellite orbital frequency where both of these frequencies are now referenced to a distant observer.

$$
\omega_{s t}^{2}=\omega_{t 0}^{2}\left(1+\phi_{c}\right) e^{-4 \phi_{c}}
$$

After taking the square root of Equation 20, expanding the result into a power series, and neglecting the higher order terms, the final formula for the geodetic precession is obtained:

$$
\omega_{s t}=\omega_{t 0} \sqrt{1+\phi_{c}} e^{-2 \phi_{c}} \cong \omega_{t 0}\left(1-3 R_{s} / 4 \rho(r)\right)
$$




\section{Comparison with experiments}

For the evaluation of the gyroscope's geodetic precession drift angle per a single orbit or per a number of orbits $N$, or per a given time period, the formula in Equation 21 is slightly modified as follows:

$$
\Delta \alpha=2 \pi N \frac{\omega_{s t}-\omega_{t 0}}{\omega_{t 0}}=2 \pi N\left(\sqrt{1+\phi_{c}} e^{-2 \phi_{c}}-1\right) \cong-\frac{3 \pi}{2} \frac{R_{s}}{\rho(r)} N
$$

The value of the physical radius $\rho(r)$ of satellite trajectory in the Earth's orbit is very close to the natural orbital radius value $r$. The difference is only a few centimeters, because the mass of Earth is relatively small and its Schwarzschild radius is only: $R_{s}=0.887 \mathrm{~cm}$. The normalized difference between the physical and natural radii is shown in Figure 1. Such a small difference will, therefore, be neglected in following calculations; however, the physical radius may play a significant role in strong gravitation fields of stars such as Pulsars and similar very compact bodies existing in the universe. The geodetic precession drift angle for the Earth's satellite orbit is then found as follows:

$$
\Delta \alpha=-\frac{3 \pi}{2} \frac{R_{s}}{R_{0}+R_{e}} \frac{365.25 \cdot 24 \cdot 60^{2} \cdot 60^{2}}{t_{0}} \frac{180}{\pi}=-6.623 \mathrm{arc} \cdot \mathrm{sec} / \text { year }
$$

where $R_{0}$ is the satellite orbit height above the Earth's surface, $R_{e}$ is the radius of Earth, and $t_{0}$ is the satellite orbital period $t_{0}=5859 \mathrm{~s}$. The result obtained from this formula agrees reasonably well with observations as can be seen in Figure 2. The West-East drift, however, is smaller than the experimental inaccuracies and is most likely caused by other factors such as the imperfect non-spherical shape of Earth rather than by the claimed frame dragging effect.

It is therefore clear that the metric in Equation 1 as well as the above described derivation of geodetic precession formula are correct. Because the metric that was used is not the GRT Schwarzschild metric, it is reasonable to expect that there might be a problem somewhere, because the two different metrics typically should not yield exactly the same results. It is also clear that this experimental result in addition of proving the correctness of the new MTG metric also proves the correctness of the relativistic formula for the Kepler's third law for circular orbits as derived earlier by Hynecek (2010). In the following section the same derivation as the one given in this section will be presented using the Schwarzschild metric.

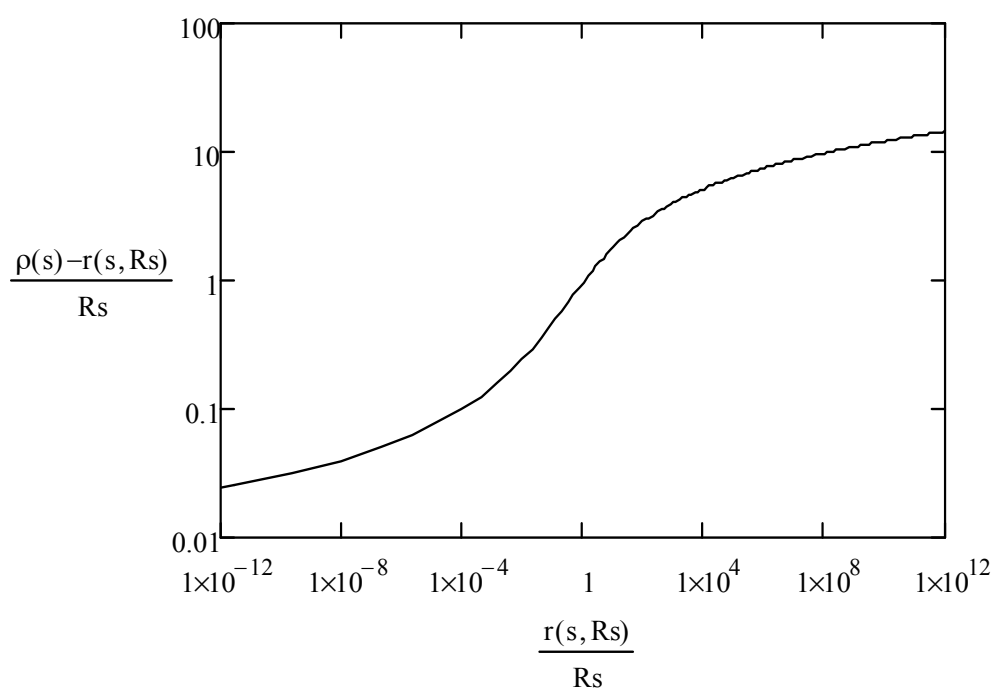

Figure 1. Normalized difference between the physical radius $\rho(r)$ and the natural radius $r$ as a function of $r$ 


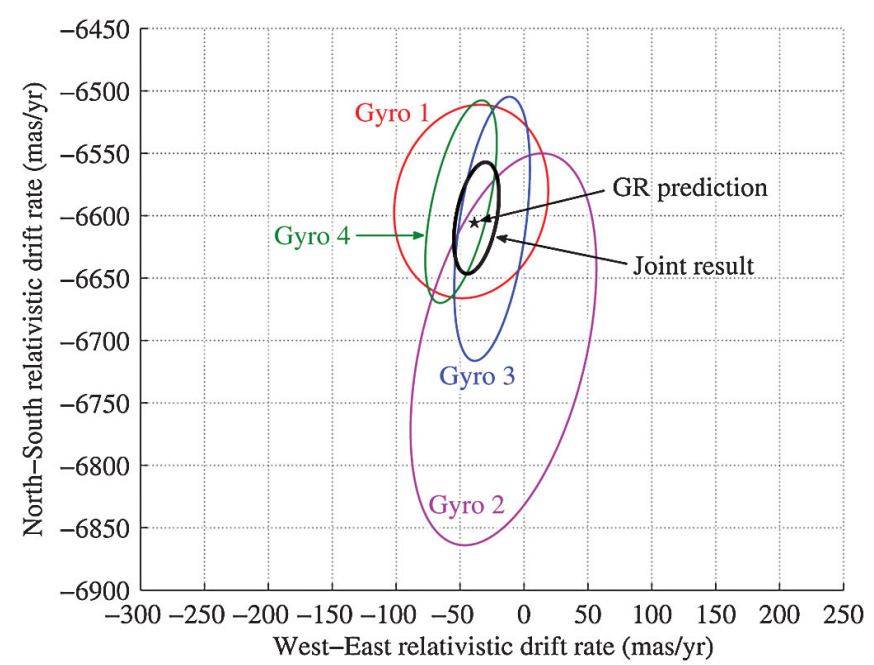

Figure 2. The Gyro's drift data from the GP-B experiment according to the final report by Everitt et al. (2011)

\section{The GRT Treatment}

The derivation presented in this section will thus start with the well known Schwarzschild metric line element:

$$
d s^{2}=g_{00}(c d t)^{2}-g_{00}^{-1} d r^{2}-r^{2} d \Omega^{2}
$$

where the time metric coefficient is equal to: $g_{00}=1-R_{s} / r$. The corresponding Christoffel coefficients that will be needed can be found elsewhere (Weinberg, 1972) and are as follows:

$$
\begin{gathered}
\Gamma_{00}^{r}=\frac{R_{s}}{2} \frac{r-R_{s}}{r^{3}} \\
\Gamma_{0 r}^{0}=-\Gamma_{r r}^{r}=\frac{R_{s}}{2} \frac{1}{r\left(r-R_{s}\right)} \\
\Gamma_{\vartheta \vartheta}^{r}=\Gamma_{\phi \phi}^{r}=-\left(r-R_{s}\right) \\
\Gamma_{r \phi}^{\phi}=\Gamma_{r \vartheta}^{\vartheta}=1 / r
\end{gathered}
$$

The component equations for the covariant spin vector derivative of the parallel transport along the satellite orbit are, of course, the same, so they do not need to be repeated here again. The second derivative of radial component of the spin vector $S$, after the substitution of corresponding Christoffel coefficients, then becomes as follows:

$$
\frac{d^{2} S^{r}}{d \tau^{2}}=-S^{r}\left(\left(1-\frac{R_{s}}{r}\right)\left(u^{\phi}\right)^{2}-\frac{R_{s}^{2}}{4 r^{4}}\left(u^{0}\right)^{2}\right)
$$

The velocity components appearing in Equation 29 can be found again by a covariant differentiation along the satellite trajectory of the velocity vector $u$ and from the Schwarzschild metric with the following results:

$$
\begin{gathered}
\left(u^{0}\right)^{2}=\frac{c^{2}}{1-3 R_{s} / 2 r} \\
\left(u^{\phi}\right)^{2}=\frac{R_{s}}{2 r^{3}} \frac{c^{2}}{1-3 R_{s} / 2 r}
\end{gathered}
$$

Similarly as in Equation 17, by using the formulas derived in Equations 30 and 31, the spin vector angular frequency $\omega_{s \tau}$ is found to be: 


$$
\omega_{s \tau}^{2}=\left(\left(1-\frac{R_{s}}{r}\right)\left(u^{\phi}\right)^{2}-\frac{R_{s}^{2}}{4 r^{4}}\left(u^{0}\right)^{2}\right)=\frac{c^{2} R_{s}}{2 r^{3}}
$$

Surprisingly, this is the classical Newton's physics formula for the frequency that corresponds to the Kepler's third law for circular orbits as observed by a distant observer even though $\omega_{s \tau}$ is still the proper angular frequency referenced to the satellite reference frame. Nevertheless, this spin vector frequency must be transformed to the distant observer reference frame as before. After the transformation according to Equation 30 the result is:

$$
\omega_{s t}^{2}=\frac{c^{2} R_{s}}{2 r^{3}}\left(1-3 R_{s} / 2 r\right)
$$

In the next step of derivation it is again necessary to find the expression for the reference orbital frequency that is needed for the normalization. The formula is found similarly as in Equation 19 using the expression for the Kepler's third law frequency: $\omega_{k t}=c u^{\phi} / u^{0}=c\left(R_{s} / 2 r^{3}\right)^{1 / 2}$. The same result is obtained using the formula derived earlier by Hynecek (2010) for the Schwarzschild metric. Both of these approaches yield the same result. This frequency is then multiplied by the gravitational red shift factor, as explained previously, to obtain the final result for the reference frequency onboard of the satellite expressed in terms of the distant observer observed satellite orbital frequency:

$$
\omega_{t 0}^{2}=\omega_{k t}^{2} \frac{\omega_{\tau}^{2}}{\omega_{t}^{2}}=\frac{c^{2} R_{s}}{2 r^{3}}\left(1-R_{s} / r\right)^{-1}
$$

However, this procedure is typically not followed by the main stream science as one can find in the published literature. It is shocking to find that the gravitational red shift factor in Equation 34 is ignored. This is very strange, no gravity induced correction factor for the time of orbiting clocks or for the oscillator frequency is included. This certainly does not seem reasonable. One can only wonder why wasn't this error found earlier by the experts who work in this field for all their professional lives? It seems that the end justifies the means in the GRT and this is not a good science as has also been discovered earlier on a related case by Hynecek (2010b). The GRT result for the normalized spin vector frequency is thus erroneously claimed to be as follows:

$$
\omega_{s t}=\omega_{t 0}\left(1-3 R_{s} / 4 r\right)
$$

Finally, from this result, the geodetic precession drift angle per a single orbit is then equal to:

$$
\Delta \alpha=2 \pi \frac{\omega_{s t}-\omega_{t 0}}{\omega_{t 0}}=-\frac{3 \pi}{2} \frac{R_{s}}{r}
$$

This is the same formula as in Equation 22 with the only difference being that the physical radius $\rho(r)$ is replaced here by the natural radius $r$. The results are thus not absolutely identical, but the difference is so small that it cannot be detected in the weak gravitational field such as the field of Earth. This formula loses its validity in strong gravitational fields for example in the field of Pulsars or similar compact objects that are found in the universe. The Schwarzschild metric exhibits a problem at the event horizon, as is well known, but this, strangely again, is not reflected in Equation 36. The formulas derived in the previous section, however, can easily extend their validity to such strong fields, because the corresponding metric does not exhibit any event horizons and Black Hole artifacts with singularities. The previously derived MTG formulas are thus more reasonable and more accurate.

The first problem, therefore, is the Kepler's third law for circular orbits derived from the Schwarzschild metric, which is identical with the Kepler's third law for circular orbits derived from the standard Newtonian physics of flat space-time geometry. The curvature of Schwarzschild space-time is not reflected in this Kepler's third law.

The second problem is the omission of the gravity induced frequency correction factor for the satellite onboard reference clocks. This factor is necessary for the spin vector frequency normalization. This omission thus seems to be a hidden error or even an intentional deception in calculations by the main stream establishment that must be there just to force an agreement between the experiment and the GRT prediction. The GP-B thus clearly confirms the metric of Equation 1 and the associated relativistic Kepler's third law for circular orbits and not the GRT metric. 
The third problem is the theoretical nonexistence proof of gravitomagnetic force and thus the frame dragging effect.

It is the author's opinion that while the GP-B experiment was very nice, thus giving its engineering very high marks for overcoming of many challenging problems, the results are not a very convincing proof of the Einstein's GRT. This is contrary to wide and enthusiastic accolades in various publications and presentations (NASA, 2008, 2011).

The new MTG metric derived previously and used by the author in this paper explains the same data with the same or ultimately a much better accuracy when the gravitational fields become strong. The GP-B results thus prove the correctness of the new metric instead of the Schwarzschild metric.

More details and a thorough explanation of the GP-B experiment can also be found in Wikipedia (2013).

\section{Generalization}

The presented derivations of geodetic precession formula can be generalized and the formula derived that is valid for any static, spherically symmetric, and orthogonal metric. This considerably shortens the calculations. The equivalent of Equation 20 is then as follows:

$$
\omega_{s t}^{2}=\omega_{t 0}^{2} g_{00} \Gamma_{\phi \phi}^{r}\left(\Gamma_{0 r}^{0}-\Gamma_{r \phi}^{\phi}\right)
$$

This, of course, leads immediately to the result for the new metric as derived in Equation 21:

and to the following result for the Schwarzschild metric:

$$
\omega_{s t}=\omega_{t 0} \sqrt{1+\phi_{c}} e^{-2 \phi_{c}} \cong \omega_{t 0}\left(1-3 R_{s} / 4 \rho(r)\right)
$$

$$
\omega_{s t(S c h)} \cong \omega_{t 0}\left(1-5 R_{s} / 4 r\right)
$$

From this formula the GRT result for the geodetic precession drift angle per orbit should be:

$$
\Delta \alpha_{(S c h)}=2 \pi \frac{\omega_{s t}-\omega_{t 0}}{\omega_{t 0}} \cong-\frac{5 \pi}{2} \frac{R_{s}}{r}
$$

This clearly shows that the GP-B test results prove that the Einstein's GRT is not the correct theory of gravity.

For the observations carried on the surface of Earth instead of by a distant observer, both frequencies $\omega_{s t}$ and $\omega_{t 0}$ undergo the same transformations, therefore, there is no difference in the results.

\section{Conclusions}

In this article it was clearly shown that the widely publicized claims of GP-B confirming the Einstein's GRT are not true. Another metric obviously exists that can be used to accurately explain the same data and it is actually this metric that the GP-B has confirmed. In addition to this fact the new metric does not predict the existence of such pathologies as the event horizons and Black Holes. This considerably adds to its credibility.

The standard procedure used for the calculation of gyroscope geodetic precession, however, relies on the Schwarzschild metric of GRT, which also provides the Kepler's third law for circular orbits that is identical to the Kepler's third law derived from the Newtonian physics of flat space-time geometry. This fact ultimately casts doubts on the correctness of the derivation of standard geodetic precession angle formula and, therefore, also on the correctness of the Schwarzschild metric, and ultimately on the correctness of the Einstein's GRT.

In conclusion the author would like to acknowledge the important role of internet and the internet journal publications such as the Applied Physics Research, which enable the unprecedented access to data and to various theories that are trying to explain them. This process is now significantly accelerated, with the information accessible to anyone who is interested. The author believes that this process will eventually lead to the abandonment of the old theories and their replacement with the new ones that describe the reality more correctly. The author also hopes that one day the nonsensical claims of Black Holes, Worm Holes, and other similar absurdities existence will reside only in the science fiction literature. Hopefully the theory of gravity will then return to a more rational state, which will be more in line with the rest of the 21 st century physics and engineering. When will this be, who knows? 


\section{References}

Everitt, C. W. F., DeBra, D. B., Parkinson, B. W., Turneaure, J. P., Conklin, J. W., Heifetz, M. I., ... Wang, S. (2011). Gravity Probe B: Final Results of a Space Experiment to Test General Relativity. Physical Review Letters, 106, 3, 221101. http://dx.doi.org/10.1103/PhysRevLett.106.221101

Hynecek, J. (2007). New Space-Time Metric, Four Tests of Gravitational Theory, and Newton's Law of Gravitation. Physics Essays, 20, 2, 313. http://dx.doi.org/10.4006/1.3119432

Hynecek, J. (2010a). Kepler's third law for circular orbits derived in metric theory of gravity. Physics Essays, 23, 3, 502. http://dx.doi.org/10.4006/1.3467821

Hynecek, J. (2010b). A note on the incorrect derivation of light deflection by a gravitating body in general relativity theory. Physics Essays, 23, 4, 588. http://dx.doi.org/10.4006/1.3495955

Hynecek, J. (2012). The Theory of Static Gravitational Field. Applied Physics Research, 4(4), 44. http://dx.doi.org/10.5539/apr.v4n4p44

Hynecek, J. (2013). The Questionable Existence of Gravitomagnetic Force. Applied Physics Research, 5(1), 28. http://dx.doi.org/10.5539/apr.v5n1p28

NASA. (2011). Retrieved from http://www.nasa.gov/mission_pages/gpb/

Synge, J. L., \& Schild, A. (1949). Tensor Calculus. University of Toronto Press, Toronto, 1949.

Weinberg, S. (1972). Gravitation and Cosmology. New York: John Wiley \& Sons.

Wikipedia. (2013). Retrieved from http://en.wikipedia.org/wiki/Gravity_Probe_B.

\section{Appendix}

The derivation of the frequency transformation formula for the frequency of oscillator that resides on the orbiting satellite to the frequency observed by a distant observer is best shown by using the Lagrangian formalism with the Lagrangian $\left(L=c^{2}\right)$ describing the satellite motion as follows:

$$
L=g_{00}\left(\frac{c d t}{d \tau}\right)^{2}-g_{\phi \phi}\left(\frac{d \phi}{d \tau}\right)^{2}
$$

The first integrals of Euler Lagrange equations of motion following from the corresponding variational principle are:

$$
\begin{gathered}
g_{00}\left(\frac{d t}{d \tau}\right)^{2}\left(1-\frac{v^{2}}{c_{\phi}^{2}}\right)=1 \\
g_{00} \frac{d t}{d \tau}=1
\end{gathered}
$$

where both velocities $v$ and $c_{\phi}$ are the velocities along the orbit defined as:

$$
\begin{gathered}
v=r \frac{d \phi}{d t} \\
c_{\phi}=c \sqrt{r^{2} \frac{g_{00}}{g_{\phi \phi}}} \approx c
\end{gathered}
$$

More specifically, Equation A5 is for the Schwarzschild metric equal to: $c_{\phi(S c h)}=c \sqrt{1-R_{s} / r} \approx c$, and for the MTG metric equal to: $c_{\phi(M T G)}=c r / \rho(r) \approx c$. The velocity of light, as is well known and according to either theory, is also not isotropic in the vicinity of gravitating bodies: $c_{\phi} \neq c_{r}$, but this effect is very small for Earth and can be neglected. From Equations A2 and A3 thus follows that:

$$
\sqrt{1-v^{2} / c^{2}}=\sqrt{g_{00}}=e^{-\phi_{c}}
$$


This equation holds always exactly true for bodies in a free fall when a correct value for the light speed, found from the metric when $d s$ is set to zero, is used in it. The correctness of this formula can be verified for the weak fields and small velocities in comparison to $c$ by squaring it and expanding the right hand side into a power series. By considering only the first two terms of expansion the well known condition for the Newtonian conservation of energy: $v^{2} / 2=\kappa M / \rho(r)$ is obtained.

In the next step, from the first integral in Equation A3, using the inverses of corresponding times, the relation between the frequency observed by a distant observer and the proper frequency onboard of the satellite is obtained as follows:

$$
\omega_{\tau}=\omega_{t} e^{2 \phi_{c}}
$$

However, there is also a Doppler effect, more precisely the transversal Doppler effect, that needs to be included:

$$
\omega_{t}=\omega_{\tau} \frac{1 \pm v / c}{\sqrt{1-v^{2} / c^{2}}}
$$

The velocity sign dependent term in the numerator will average out over the satellite's orbit and after the substitution for the velocity from Equation A6 the following formula results:

$$
\omega_{\tau}=\omega_{t} e^{-\phi_{c}}
$$

The same result without averaging out the linear term in the numerator is obtained when the distant observer is positioned on the axis perpendicular to the satellite's orbital plane.

By combining the results form Equations A7 and A9 the final formula for the frequency transformation from the orbiting satellite proper frequency $\omega_{\tau}$ to the distant observer observed frequency $\omega_{t}$ is:

$$
\omega_{\tau}=\omega_{t} e^{\phi_{c}}
$$

This result is used in Equation 19. It is interesting to note that this formula is identical with the frequency transformation formula that would be obtained for an equivalent stationary oscillator or clocks positioned at the same potential of the gravitational field that exists at the satellite's orbit. The formula can be derived from the relation: $d \tau=\sqrt{g_{t t}} d t=e^{-\phi_{c}} d t$, and it is the well known gravitational red shift formula:

$$
\omega_{\tau}=\omega_{t} e^{\phi_{c}}
$$

While this finding is interesting it is not surprising, because the free fall, which the motion of the satellite represents, is equivalent to the action of gravity in a stationary reference frame.

One last comment is necessary, however, which is related to the definition of the distant observer. The meaning of the distant observer is the observer that is just removed from the gravitational field, not necessarily an observer placed in a particular distant coordinate location. 